\title{
Art-therapeutic techniques and methods for working with children of preschool and primary school age
}

\author{
N.M. Tolkova ${ }^{1 *}$, and T.N. Morozova ${ }^{2}$ \\ ${ }^{1}$ State Educational Institution of Higher Education of the Moscow Region State Humanitarian and \\ Technological University of Orekhovo-Zuevo, Orekhovo-Zuevo, Russia \\ ${ }^{2}$ State Educational Institution of Higher Education of the Moscow Region State Humanitarian and \\ Technological University of Orekhovo-Zuevo, Orekhovo-Zuevo, Russia
}

\begin{abstract}
The article reveals a topical topic of research into the influence of specially organized art-therapeutic techniques and techniques on the level of emotional self-regulation of the personality of children of preschool and primary school age. The need to study it is due to the possibility of emotional, creative self-expression of children in the classroom with the use of art therapy techniques and techniques. The study presents theoretical and practical methods. The results of work with teachers in this direction with children of preschool and primary school age are presented.
\end{abstract}

\section{A problem statement}

The relevance of studying art therapy techniques and techniques in working with children is beyond doubt. Currently, there is a rapid development of art therapy in Russia. In preschool and primary education, art therapy involves a combination of visual creativity with musical activity, role-playing game, as well as various forms aimed at the creative self-expression of children.

Research on art therapy in pedagogy and psychology is reflected in the works of foreign and domestic scientists. The works of foreign teachers and psychologists indicate the important role of art in the education of children: E. Seguin, J. Demor, O., Decrolie [13, 17], as well as domestic educational psychologists L.S. Vygotsky, A.I. Grabov, V.P. Kashchenko and many others. others [5].

Many teachers, psychologists, and artists have considered the art therapy area of activity. The founders are Z. Freud and K.G. Jung, they argue that drawing is the result of unconscious mental processes [7,8]. In the works of A. Maslow, R. May and K. Rogers, one can find a statement that the development of creative potential reduces nervous tension and is aimed at combating internal conflicts [12].

In scientific research of domestic teachers involved in the study of the artistic education of children, N.I. Sakulina, N.A. Vetlugina, V.A. Ezikeeva, T.S. Komarova et al. Revealed

${ }^{*}$ Corresponding author: ipcs-profped@yandex.ru 
that the process of art perception is a complex mental activity that combines emotional, cognitive developmental processes. The artistic activity of children is directly related to the formation of sensory standards in preschool children [5].

The founder of the Russian school of art therapy can be considered A.I. Kopytina.

Researchers of art therapy techniques and techniques have confirmed the positive impact of art on preschool and primary school children.

\subsection{The objective of the work}

There are many approaches to the upbringing of children's artistic and aesthetic abilities. The basis of our research was the provisions on art as a factor in the psychological and pedagogical development of children in the works of foreign scientists H. Reed, E. Kramer, etc., in Russia this problem was dealt with by E.I. Bruno, E.I. Karabanova, G.V. Burkovsky who assert in their works that visual activity helps to express one's thoughts without words and, in addition, the products of visual activity (drawing, modeling, etc.) contribute to the internal development of mental processes. During the research, we applied theoretical methods such as generalization, classification, systematization $[1,3,6,9]$.

Practical points of view were considered at international and interregional conferences: "Art therapy techniques and techniques in working with children of preschool and primary school age", "Artistic and aesthetic development of children of preschool and primary school age", etc., as well as in publications in periodicals with the results of psychological and pedagogical experience.

\section{Materials and the results of the research}

In modern society, transformation is constantly taking place aimed at transformation in private industries such as pedagogy, art, medicine, etc. which leads to the emergence of border industries - educational psychology, curative pedagogy, art pedagogy, etc.

In connection with the orientation towards a new educational paradigm for the development of a culturally-oriented personality in modern pedagogy, the direction of artistic culture has received the greatest interest. So, Nikolai Aleksandrovich Berdyaev gives a clear definition of the place of the child in the modern cultural space, pointing out that the child is the main value and object of culture.

Lev Semenovich Vygotsky noted that "in terms of its inner content, the process of a child's cultural development can be characterized as an activity aimed at developing a child's personality and worldview." the importance of the formation of the child's personality through art through various types of artistic and creative activities is great. In his writings, Vygotsky assigned a large role to the study of the child's cognition of the inner world [5].

In modern society, educators and psychologists have a continuing interest in the impact of the arts on the development of children in the process of education and upbringing in kindergarten and primary school.

Pedagogical science has undergone various transformations and modifications, combining several directions and now art pedagogy and art therapy are widely used. Both of these concepts consist of two parts, the first is art - translated from English as "art", the second part of these concepts is different, in one case pedagogy is the science of education, training and development, in the other - therapy - a medical term meaning a therapeutic effect.

The scientific foundations of modern pedagogical art therapy were developed by Lydia Anatolyevna Ametova, Lydia Dmitrievna Lebedeva, etc. In their works, they consider art in several directions: as a factor in the development and formation of personality, as well as a 
means of preserving the child's psyche from aggressive environmental influences, a means of preserving health of the younger generation $[3,10,11,13,14]$.

Art therapy techniques and techniques are actively used in the context of interaction with children of preschool and primary school age, since their implementation is possible in various forms (group, individual). Using techniques and techniques of art therapy in the educational process, the teacher solves specific educational tasks, which are fixed in the process of interacting with a preschooler or a younger schoolchild by means of the arts $[15$, $16,17]$. In addition, the child has the opportunity to express his inner experiences, thoughts, not only in a verbal way, but also in a non-verbal way (body plastic, emotions, etc.).

The modern direction of art therapy includes a large number of areas and technologies, we concluded that only a systematic and complex impact on children leads to positive results.

The following areas of art therapy are widely used:

1. Fairytale therapy - a method used to develop creativity, attention, fine motor skills of children through a fairy tale. In the preschool educational institution of the Moscow region, a series of books, therapeutic fairy tales, "Tales of Mom Mouse", is widely used.

2. Sand therapy is an affordable method of helping a child grow up as a healthy, harmoniously developed personality. Classes with sand are held both in special classes and can be used in the free play activity of children. With the help of sand therapy, the child develops motor skills (large and small), imagination, can open up emotionally, etc. Classes with sand are conducted by: preschool teachers, elementary school teachers, psychologists, defectologists, etc.

3. Kinesitherapy - dance movement direction. Through dance, movements, the child reveals his inner world to others. All activity is based on the relationship: the brain, emotions, movement. This technique is effective from an early preschool age. The elements of the methodology are introduced into the theatrical activities of preschool children, are actively used by speech therapists in group and individual lessons, as well as by physical education instructors.

4. Puppet therapy is a method of knowing oneself through playing life situations with dolls. With older children, preparatory to school age, as well as with younger schoolchildren, we use making dolls ourselves, playing life situations.

5. Isotherapy is the most accessible and popular technique that uses methods and techniques of visual activity (drawing, modeling, drawing, etc.). It is one of the fundamental techniques of art therapy.

6. Bibliotherapy - a method of healing with books. Based on identifying yourself with a positive hero. It means reading fiction (specially selected, conducive to moral and positive psychological development), corresponding to age.

7. Color therapy is a technique aimed at correcting the psycho-emotional state of a child using certain colors.

The art therapy direction in the creative development of children is used by teachers and psychologists for psychological and pedagogical correction of emotional states. The purposeful introduction of art-therapeutic techniques and techniques into the educational process acts as a method of psychological and pedagogical correction and is the most effective means of corrective influence on the emotional sphere of the child, helps to find a way out of the child's internal conflicts, strong emotions, contributes to the awareness of internal experiences, sensations and feelings.

Our team carried out a study of the influence of purposeful art-therapeutic techniques and techniques on the level of emotional self-regulation of the personality of preschool and primary school children.

We suggested that there is a relationship between the level of emotional self-regulation and the use of techniques and techniques of art therapy in the educational process. Work in 
this direction provided for the creation of special psychological and pedagogical developmental programs. A quasi-experiment was carried out.

The work took place in several stages. At the initial stage, our team prepared a catalog of psychological and pedagogical developmental classes aimed at achieving the intended goals and the task of the study, systematized the practical material of preschool educational organizations and primary secondary schools of the Moscow region. The content of psychological and pedagogical developmental classes and the choice of the necessary methods of art therapy were determined.

The study was carried out at several sites: Municipal budgetary preschool educational institution "Svetlyachok", Noginsk, Municipal autonomous preschool educational institution "Kindergarten" Fidgets", Balashikha, Municipal autonomous educational institution "Davydovskaya gymnasium“, Likino-Dulyovo.

We have conducted a study providing for the creation of a catalog of psychological and pedagogical developmental classes. The package of techniques used by us to diagnose the emotional state of pupils included the following techniques:

1. "Fears in the houses" methodology Zakharov A.I. and Panfilova M.A.

2. Graphic technique "Cactus" by M.A. Panfilova.

3. Luscher color test.

Luscher's color test made it possible to determine that $66 \%$ of pupils had an average anxiety level, $28 \%$ had a high level and $6 \%$ of all studied children had a low level of anxiety. The results are shown in Figure 1.

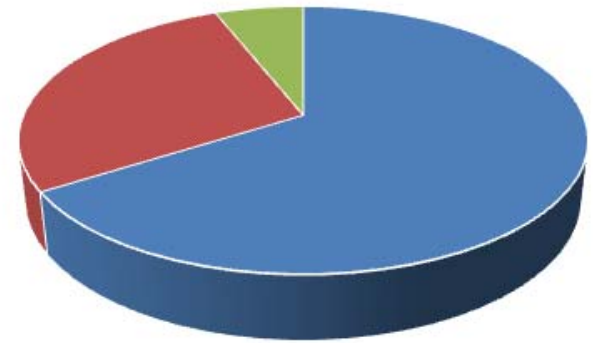

- average level of anxiety - low level of anxiety

- high level of anxiety

Fig. 1. Level of anxiety (Luscher's color test).

Thus, in the diagram, we see that $94 \%$ of children have a level of anxiety above the average, which is evidence of the low emotional fitness of the subjects.

Methodology "Fears in the house" Zakharova A.I. and Panfilova M.A. allowed us to identify the number of fears among pupils and their nature. The analysis of the obtained results revealed that in $70 \%$ of the subjects fears prevail, in $30 \%$ of the subjects at the level of the age norm. The result is shown in Figure 2. 


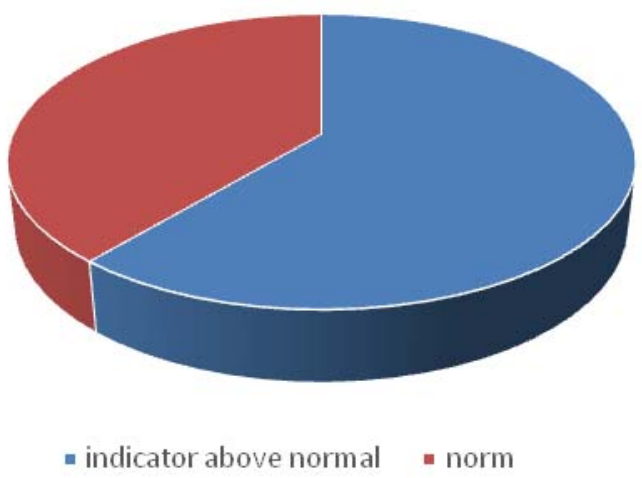

Fig. 2. The number of fears among students and their nature (Methodology "Fears in the house" Zakharova A.I. and Panfilova M.A.).

Thus, the diagnostic technique made it possible to reveal that in most of the subjects the number of fears exceeds the age norm.

When carrying out the graphic technique "Cactus" by M.A. Panfilova, we identified the emotional state of each child, and also revealed the presence of aggression and its intensity. The analysis of the results was grouped according to characteristic features and amounted to aggression in $55 \%$ of the subjects against the background of overestimated self-esteem, $35 \%$ of the pupils showed aggression against the background of low self-esteem, and only in $10 \%$ of the subjects the aggression was at the level of the age norm and manifested itself as a protective mechanism of the child's psyche. The result is shown in Figure 3.

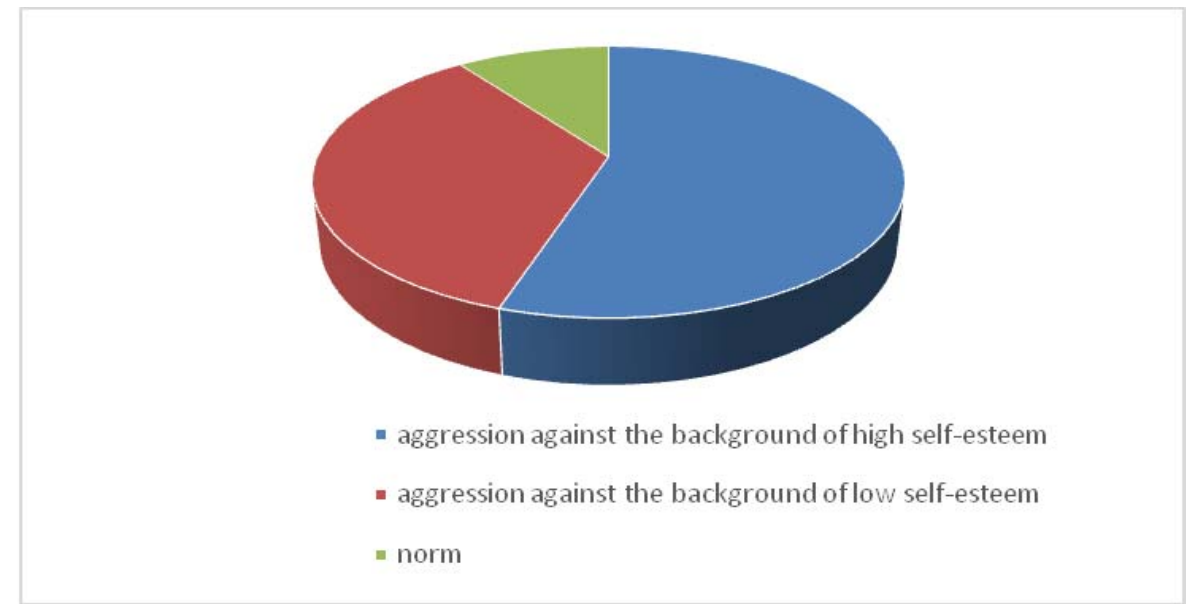

Fig. 3. Agreya and its intensity (graphic technique "Cactus" by M.A. Panfilova).

Thus, the performed method allowed us to reveal the presence of aggressive behavior in a larger number of subjects, which can lead to a negative attitude with the collective of peers.

Psychological and pedagogical activity was based on the goals and objectives of our research.

Purpose of the study: creation of the necessary conditions in the educational space for mastering the skills of emotional self-regulation using techniques and means of art therapy. 
Tasks:

1. Overcoming negative experiences.

2. Reducing the level of anxiety.

3. Development of the ability to interact with a peer.

4. Develop an understanding of your emotional state and peers.

5. Teaching self-regulation techniques.

The study was based on the basic general pedagogical principles:

1. The unity of diagnostic and correctional and developmental tools.

2. Taking into account the age, psycho-physiological and individual characteristics of the subjects.

3. Consistency.

4. The complexity of psychological, pedagogical, developmental impact on children.

The experiment involved the creation of a catalog of psychological and pedagogical developmental classes aimed at emotional self-regulation of preschool and primary school children. The group of subjects included 40 children of five years old and 30 children of eight years old. The study took place during the school year. The work was carried out in a group form, the following techniques and techniques were used to implement the tasks:

1.Non-traditional drawing methods are used with preschool children:

- drawing with fingers, palms;

- drawing on wet paper;

- drawing with leaves;

- paper plastic;

- testoplasty, etc.

2. In working with children of primary school age, non-traditional methods of visual activity are used:

- tamponing;

- drawing on wet paper - isothread, monotype, blotting;

- scratchboard, etc.

3. Game therapy.

4. Music therapy.

5. Dance therapy.

In order to determine the effectiveness of the program aimed at the emotional selfregulation of children through the use of art therapy techniques and techniques, we conducted a quasi-experiment ascertaining. The purpose of which was to confirm the hypothesis of the study, to clarify the methods, techniques and techniques of educational activities.

At the stage of the control section, the same techniques were used to diagnose the subjects ("Fears in the house", the graphic technique "Cactus" and the Luscher color test). At this stage, significant positive changes were revealed in the emotional self-regulation of preschool and primary school children.

Luscher's color test on the control section revealed that $40 \%$ of the pupils have an average level of anxiety, $12 \%$ have a high level of anxiety. The low level of anxiety was $48 \%$ of all studied children. A comparative analysis of this technique at the diagnostic and control stages gave positive dynamics in the direction of relieving anxiety in the subjects.

Control slice according to the method "Fears in the house" Zakharova A.I. and Panfilova M.A. found that fears prevail in $40 \%$ of the subjects, and in $60 \%$ of the subjects at the level of the age norm. In a comparative analysis with the initial diagnostic result, we can conclude that we have achieved positive dynamics in this direction.

The control section using the "Cactus" method also gave a positive result and revealed that $25 \%$ of the subjects showed aggression against the background of high self-esteem, $25 \%$ of the pupils showed aggression against the background of low self-esteem, and only 
$50 \%$ of the subjects showed aggression at the level of the age norm and manifested itself as a protective mechanism of the child's psyche. After analyzing the primary diagnosis and control section, we can conclude that the indicators in each direction have a significant positive trend.

Our team carried out a comparative analysis of the results, which made it possible to conclude that a series of psychological and pedagogical developmental classes, as well as the observance of all the above conditions, made it possible to significantly reduce negative emotions in the subjects and increase the level of emotional self-regulation of pupils. implementation of the planned activities in the direction of the influence of targeted arttherapeutic techniques and techniques on the level of emotional self-regulation of the personality of children of preschool and primary school age.

\section{Conclusions}

Thus, we can conclude that the research carried out within the framework of the presented topic made it possible to study the specifics of the introduction of methods and techniques of ATR therapy in modern preschool educational organizations and primary schools.

Based on the results of the research work, the following conclusions were made:

1. The introduction of art therapy techniques and techniques in the educational process leads to a positive emotional disclosure of the child.

2. Techniques and techniques of applied technologies are available, understandable and valid.

3. The level of the educational process goes to a higher quality level.

Work on this topic continues, we are making qualitative and quantitative changes in psychological and pedagogical developmental classes, new technologies are being introduced for art-therapeutic influence on children of preschool and primary school age.

\section{References}

1. Compiled by Yu.O. Krasniy, The ABC of Feelings: A program for the complex aesthetic development of preschool children and primary school children, p. 32 (Moscow: Education, 1993)

2. L.A. Ametova, Formation of art therapy culture of junior schoolchildren. "Himself an art therapist", p. 36 (Moscow: Moscow State Open Pedagogical University, 2003)

3. A. Asmolov, Psychology, art, education, Art in school, 6 (1993)

4. L.S. Vygotsky, Childhood learning and development, p. 305 (Moscow, 2006)

5. L.D. Lebedeva, The practice of art therapy: approaches, diagnostics, training system, p. 256 (St. Petersburg: Rech, 2003)

6. K.G. Jung, Selected Works Carl Gustav Jung, p. 288 (Moscow: Publishing house of the Russian Christian Humanitarian Academy, 2014)

7. M.S. Kagan, Philosophy of culture, p. 300 (St. Petersburg, 1996)

8. G.M. Kodzhaspirova, History of education and pedagogical thought: tables, diagrams, basic notes, p. 145 (Moscow, 2003)

9. A.I. Kopytin, Theory and methodology of art therapy, p. 366 (St. Petersburg: Peter, 2020)

10. M.V. Kiseleva, Art therapy in working with children: a guide for child psychologists, teachers, doctors and specialists working with children, p. 280 (St. Petersburg: Rech, 2008.) 
11. A. Maslow, Motivation and personality, p. 480 (St. Petersburg, 2002)

12. P.I. Pidkasisty, Zh.S. Khaidarov, Game technology in learning and development, $\mathrm{p}$. 269 (Moscow: Moscow Pedagogical University, 2006)

13. O. Sukhinina, Formation of the child's personality in the process of acquaintance with art, Preschool education, 2, 36-38 (2004)

14. N.M. Tolkova, Art therapy methods and techniques in working with young children with disabilities in a preschool organization, Problems of modern pedagogical education, Ser.: Pedagogy and Psychology, Collection of scientific works, 62 (2), 233234 (Yalta: RIO GPA, 2019)

15. N.M. Tolkova, The relationship between the specificity of communication and the age characteristics of young children, Bulletin of the Vladimir State University named after Alexander Grigorievich and Nikolai Grigorievich Stoletovs, 22 (41), 129-136 (Vladimir, 2015)

16. R. Frager, J. Feydiman, Personality, Theories, exercises, experiments (PrimeEuroznak, 2004)

17. J. Tuillier, Edouard Seguin (1812-1880), pioneer of child psychiatry (2006) 